\title{
Mental health literacy and the anxiety disorders
}

\author{
Adrian Furnham*, Chiara Lousley \\ Research Department of Clinical, Educational and Health Psychology, University College London, London, UK; \\ *Corresponding Author: a.furnham@ucl.ac.uk
}

Received 23 January 2013; revised 25 February 2013; accepted 9 March 2013

\section{ABSTRACT}

Context: This study set out to investigate the mental health literacy (MHL) about eight anxiety disorders (ADs), using vignette methodology. Methods: In all 317 British Adult participants completed a questionnaire with vignettes describing eight anxiety disorders including OCD, PTSD, Agoraphobia, Specific Phobias, Social Phobia, Separation Anxiety Disorder, GAD and Panic Disorder. Recognition, beliefs about treatments and perceived life adjustment (happiness, success at work) of specific people with these disorders were assessed. Results: Literacy levels varied across anxiety disorders, with high recognition of OCD $(64.67 \%)$, but very poor for panic disorder (1.26\%), GAD (2.84\%) and separation anxiety disorder (5.99\%). There were few significant effects of vignette gender on literacy levels. MHL for most anxiety disorders was relatively low; particularly panic disorder, GAD and separation anxiety disorder. Social Phobics were judged as least happy and adjusted and agoraphobics least successful at work and would benefit most from psychological help. Conclusions: Overall recognition of the anxiety disorders was poor. Beliefs about adjustment and treatment varied widely as a function of each disorder. Implications of the research for education of the public and limitations of this research are considered.

Keywords: Anxiety Disorders; Gender; Vignette; Mental Health Literacy

\section{INTRODUCTION}

\subsection{Mental Health Literacy}

This paper is concerned with the mental health literacy (MHL) specifically about the anxiety disorders (ADs). The term MHL is defined as "knowledge and beliefs specifically about mental disorders which aid their recognition, management or prevention" [1]. MHL encom- passes several aspects; including recognition of disorders and attitudes facilitating this and help-seeking behaviour [2]. It has attracted a great deal of interest over the previous decade. The research has gone in two directions: first, to look at MHL for very specific disorders like schizophrenia [3], depression [4] or the conduct disorders [5] and second, to look the MHL of certain groups like young people [6-8].

A plethora of work has been conducted into the MHL of depression and schizophrenia [4]. Recognition of depression is found to be very high, with schizophrenia close behind $[2,3]$. The majority of studies report successful recognition as "correct" labelling of a disorder; defined as using the currently accepted psychiatric terminology. Using vignette methodology it was found that $75.6 \%$ "correctly" recognised depression [4]; similarly "correct" identification for depression has been reported at $97.2 \%$ and schizophrenia at $61 \%$ [9]. Through reporting on treatment beliefs, GP's or family doctors were found to be considered the best help for depression [4]. Worryingly, over $43 \%$ of participants considered the cause of depression to be weakness of character, which could indicate why, despite a high recognition of the disorder, little is still known about its cause and which may affect help-seeking patterns. However, $73.5 \%$ thought the cause of depression to be genetic and $90 \%$ due to childhood abuse.

\subsection{Anxiety Disorders (ADs)}

Research into the MHL concerning any or all of the ADs seems scarce. ADs include generalised anxiety disorder (GAD), panic disorder (with and without agoraphobia), obsessive compulsive disorder (OCD) and posttraumatic stress disorder (PTSD) (APA DSM-IV-R).The new proposals for the DSM-5 due to be published in 2013, have separated agoraphobia as a distinct disorder separate from panic disorder. The criteria have also proposed to remove OCD and PTSD from anxiety disorders into their own separate class of disorders [10]. However, in terms of this paper, OCD and PTSD will be included due to their current diagnosis as anxiety disorders.

ADs are amongst the most common mental disorders, 
with prevalence rates ranging from $13.6 \%$ and $28.8 \%$ in Western Countries [11]. An overview of results has been provided from 14 mental health surveys including one which reported anxiety disorders as the most prevalent class of disorders at $18.1 \%[11,12]$. These studies also report that specific phobias and social phobia (or social anxiety disorder) are the most common of anxiety disorders. Similarly reports show that in US adolescents specific phobia, social phobia and separation anxiety are the most common disorders; and research reports an even higher prevalence rate of $39.1 \%$ for anxiety disorders overall [13].

A few MHL studies have focused on very specific anxiety disorders, such as one which investigated social phobia and PTSD recognition rates, not taking into account the other anxiety disorders [14]. Using phone interviews rates of recognition for PTSD were reported to be similar to that of schizophrenia (with around $1 / 3$ providing the "correct" label) but only $9.2 \%$ of participants "correctly" labelled social phobia; a much lower rate. Participants more commonly used labels such as anxious, shy and low self-confidence for social phobia; from which the researchers concluded they were less likely to see it as a mental disorder.

Knowledge of PTSD has been compared to depression using vignette methodology [15]. This study found a significant difference between PTSD and depression in terms of a "correct" differential diagnosis $(67.5 \%$ to 94.4\%), "correct" prescription and treatment options. Similarly the prevalence of PTSD was found to be underestimated by GPs [16], and rather than referring to the preferential treatment of psychological therapy most medicated patients used SSRI's (serotonin-specific reuptake inhibitors). Both papers suggest even in primary care with trained professionals there are some gaps in mental health literacy for PTSD, particularly in concern with treatment beliefs, which would imply the general public's knowledge for PTSD treatment would be even worse since they do not have training.

Some looked at MHL of the anxiety disorders, looking at social phobia, GAD, Panic Disorder and OCD [17]. Using vignette methodology the researchers asked three questions. Results showed variability in "correct" labelling, with depression, OCD and social phobia all "correctly" assigned their label by greater than $86 \%$ of participants, whereas panic disorder was assigned by $47.7 \%$ and GAD by $41.5 \%$. Other than panic disorder, the most popular assigned label was "medical problem" (27.6\%), and for GAD $41.4 \%$ chose "general life stress" to label the problem. From this the authors concluded OCD and social phobia were recognised at similar rates to that of depression. This finding contradicts other research [14] which found poor recognition for social phobia, but this could be due to both very different methodologies and samples. A limitation of some of the research, which may have explained the high recognition for social phobia, was the use of a list of labels for the participant to choose from; this may have inflated recognition rates compared to an open-ended question being used [17]. Moreover, beliefs on whether the person should seek professional help varied across disorders and were dependent on symptom attribution; with a higher proportion suggesting treatment if they thought the problem was caused by mental illness compared to factors such as stress or personal weakness.

\subsection{Current Study}

The current study attempts to study MHL about the ADs in a population of young people. One issue regarding MHL which has not yet been investigated is whether the gender of the character described in the vignette (to be known as "vignette gender") affects MHL. With anxiety disorders in particular, there is a much higher prevalence amongst women than men [18]. It was found that more women met the criteria for an anxiety disorder in their lifetime, with one in three women compared to $22 \%$ of men. It was also found anxiety disorders are more disabling in women than men; measured by a greater illness burden (number of doctor visits and days missed off work). The research did not find any significant gender differences for social phobia, though some have found a significantly higher rate in women [19]. Similarly, when examining the after-effects of earthquakes female gender was found to be a significant risk factor for developing PTSD [20]. This finding was corroborated by researchers who found PTSD 10 months after an earthquake in $51.7 \%$ of females compared to $25.7 \%$ males in a population of students [21]. This higher prevalence in females suggests that people would recognise anxiety disorders better in females than male vignettes.

Past research has examined what factors predict levels of MHL including age, education level and topics studied, gender and contact with particular patient groups [7]. Numerous studies have found female gender is a significant predictor of MHL $[6,22,23]$ though results remain equivocal with not all studies finding a sex difference in MHL.

Studies have examined the importance of a good level of MHL, particularly focusing on how this often leads to greater help-seeking. "Correct" labelling of mental disorders has been found to predict greater levels of appropriate help seeking behaviour [24,25]. Others found a diagnosis of an affective or anxiety disorder was given by $93 \%$ of GP's when patients presented psychologising attributions, compared to only $23 \%$ when presenting somatising attributions; thus showing symptom attribution as a significant predictor of diagnosis [26]. 
Given the previous literature it was predicted that the recognition, treatment recommendations and perceived adjustment would vary significantly across the anxiety disorders described (H1). Next, that there would be a significantly higher proportion of "correct" labelling and sympathy for vignettes with female characters (H2). Finally it was predicted that females would demonstrate significantly higher mental health literacy than males (H3).

\section{METHOD}

\subsection{Participants}

Of the 317 participants in the current study, 103 were first year Undergraduate Psychology students at the beginning of their course (and who had received no lectures on clinical psychology or the anxiety disorders) and 214 were non-students recruited from the general (London) population using opportunity sampling. 51.7\% were female, with a mean age of 35.65 years, ranging from 18 80 years (SD 16.98 yrs). $63.4 \%$ of participants were British White, 23.7\% Asian, 1.6\% African-Caribbean and $11.4 \%$ other. In terms of marital status; $32.8 \%$ were married, 4.4\% cohabiting, $47.3 \%$ single, $6.6 \%$ divorced, $3.2 \%$ widowed, $5 \%$ reported "other" and the remaining $0.6 \%$ did not report. $11.7 \%$ of participants had been treated for a (minor) psychiatric illness, but were fully recovered, and $65.6 \%$ reported having personal experience of knowing someone with a mental illness.

In the non-students group, $27.6 \%$ held a secondary school certificate as their highest qualification, 22.45 held a BA or BSc, $21.5 \%$ held "other higher qualification" (e.g. a-level $/ 12^{\text {th }}$ grade or equivalent), $16.8 \%$ held an MA, MSc or $\mathrm{PhD}, 3.7 \%$ held only a school certificate $\left(10^{\text {th }}\right.$ grade), $3.7 \%$ were in full time education (e.g. an internship/army training/work-related courses), 3.3\% held no academic qualifications and the remaining $0.9 \%$ chose not to report their qualifications. $87.4 \%$ of the non-student group had not studied Psychology formally at any point.

\subsection{Anxiety Disorders Questionnaire}

The questionnaire consisted of 8 vignettes describing the anxiety disorders; OCD, PTSD, GAD, specific phobia, social phobia, panic disorder, agoraphobia and separation anxiety disorder (available from the second author). The vignettes were selected from different sources; the vignettes for GAD, panic disorder, social phobia and OCD were taken from Coles and Coleman's (2010) paper and the PTSD vignette was taken from Munro et al. (2004). The remaining vignettes were adapted from books: the agoraphobia vignette [27]; the separation anxiety disorder vignette [28]; and the vignette for specific phobia [29]. These were evaluated by two clinical psychologists, experts in the anxiety disorders, as face valid. Because most had been used in previous studies it is assumed that they have both content and construct validity. [7]

Each vignette was adapted to have both male or female persona, with all other characteristics and descriptions in the vignette remaining the same. There were thus two versions of the questionnaire; each participant got either one randomly with half the vignette characters as male or female. They were around 90 - 150 words long and written in English. An example for the female version of agoraphobia is given:

Celia, a 29-year-old female has fears of being in public places such as supermarkets, buses or trains in case she has an attack of anxiety and collapses. Since they began seven years ago, the symptoms have waxed and waned. At present she is housebound unless accompanied by her husband or friends. Even when accompanied she sometimes becomes anxious and may panic. She worries considerably before each outing.

After each vignette participants were asked 9 questions about the character to measure the aspects of mental health literacy. The first being an open ended question asking "What, if anything, would you say is X's main problem?" Responses to this question were then coded into "yes" or "no" dependent on whether they provided the "correct" name of the disorder; the use of the currently accepted Psychiatric terminology. The responses were also coded into categories, in order to establish the most common labels given for each disorder. A second coder was used to ensure the reliability of coding.

The following 8 questions used 7-point Likert scales. For questions $2-7$ the scale consisted of $1=$ not at all to 7 = extremely. Questions 2 and 3 asked participants how distressing and difficult to treat they thought the problem would be, and question 4 how sympathetic they were towards the person described. Questions 5 to 8 asked about how well adjusted they felt the person was; including ratings of happiness (Q5), success at work (Q6) and personal relationships (Q7). The higher the rating (closer to 7) the better adjusted the participant thought the person was. A total adjustment score was also calculated by taking the average of the three questions. Question 8 asked on a scale of $1=$ not at all to $7=$ definitely, whether the person should seek help for their problem. Question 9 provided participants with a list of treatment options; none, friends, parents, other family members, GP, Psychologist/Psychiatrist, books and internet. Participants had to rate on a scale of $1=$ not very likely to 7 $=$ very likely for each option as a possible source of treatment.

\subsection{Procedure}

Ethical approval for the study was sought and received 
from the departmental committee. Participants were recruited by paid post-graduate students mainly in public places (railway stations, coffee bars) whose task was collect 300 participants of mixed, sex, age and ethnic background. Around 30\% refused the invitation primarily because of time constraints. Participants were given an instruction sheet and provided informed consent. Questionnaires were administered on paper, and took approximately 15 minutes to complete. After finishing participants were debriefed with a debriefing form and thanked for their participation. They were not remunerated for their participation.

\section{RESULTS}

\subsection{Inter-Rater Agreement on Coding of Labels}

Reliability analyses involved a secondrater (postgraduate) coding $10 \%$ of questionnaires using the same coding system as the author (correct/incorrect). Results demonstrated excellent reliability: Kappa 0.81 .

\section{Vignette Recognition of Anxiety Disorders}

Figure 1 shows the anxiety disorders in descending order for "correct" labelling; use of the current Psychiatric terminology. It is evident that the ability to name a disorder varies, with high levels of recognition of OCD compared to very poor recognition of panic disorder, GAD, separation anxiety disorder and social phobia. Chi-square tests revealed that for all disorders, the proportion of participants "correctly" or "incorrectly" labelling the disorder was significantly different $(p<0.004)$. In all cases the majority gave "incorrect" labels, except OCD where the majority "correctly" labelled the disorder. Moreover, in order to assess the first hypothesis and test whether recognition ("correct" labelling) differed between the disorders, a Cochran's Q test was used. This revealed a significant difference in the proportion of vi-

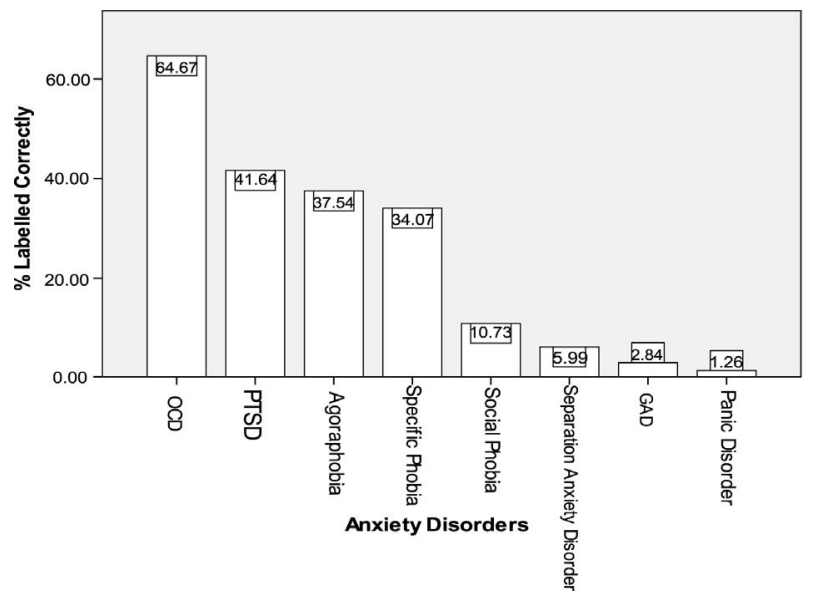

Figure 1. A graph illustrating the percentages of "correct" labelling across the anxiety disorders. gnettes "correctly" named, $Q(7)=672.50 p<0.001$; therefore showing recognition rates varied significantly as a function of the disorder type. This confirms $\mathrm{H} 1$.

Tables 1(a)-(h) demonstrate the variety of responses given for each disorder when asked what the characters main problem was. For many disorders, major symptoms were listed such as for agoraphobia $10.1 \%$ said the person had a "phobia/anxiety of public places". For panic disorder $27.8 \%$ gave the label of "panic attacks" which is a major symptom. Tables 1(a)-(h) also show that "fear" was a common label given for many of the disorders, as was "anxiety".

\subsection{Treatment Beliefs Vary across Disorders}

To compare whether participants ratings for whether the character should seek help for their problem differed across anxiety disorders (the second part of the first hypothesis), a one way repeated measures ANOVA was used. Mauchly's test of sphericity was significant ( $W=$ $0.56, p<0.001$ ), therefore Greenhouse-Geisser estimates were taken to correct for degrees of freedom. The results suggested that participants rating for seeking help differed significantly across anxiety disorders; $F(6.08$, 1922.63) $=40.58, p<0.001$. A Post-hoc, pair-wise comparison showed this to be the case. It is apparent from the graph that there were essentially three groups which differed significantly from each other: Agoraphobia and PTSD did not differ from each other and were judged as most in need to help; followed by four Ads that did not differ from one another (OCD, Separation Anxiety Disorder, Panic Disorder and Social Phobia) while the final two (GAD and specific phobia) which did not differ from each other, but were significantly different from all others on this rating.

Figure 2 demonstrates the variation in ratings; with PTSD given the highest rating for seeking help and spe-

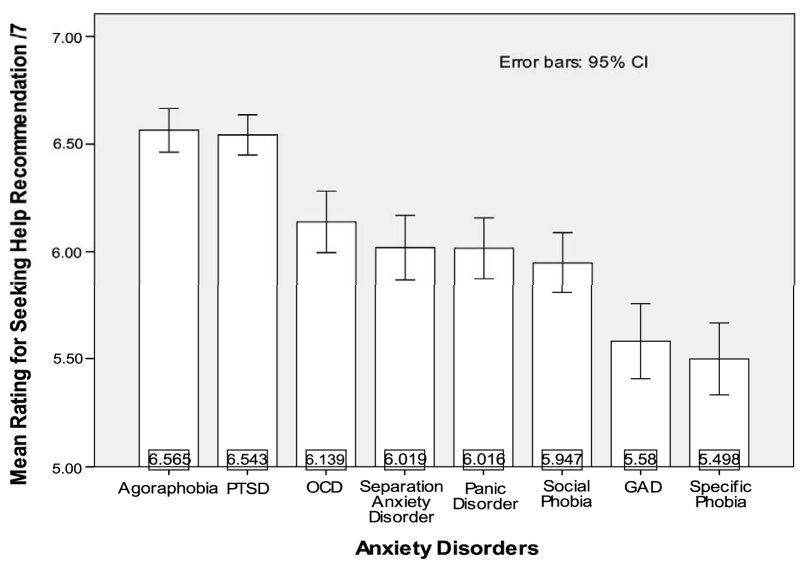

Figure 2. A graph illustrating the mean ratings for seeking help recommendations across the anxiety disorders. 
Table 1. (a) Ranking of Labels of Panic Disorder; (b) Ranking of Labels of Agoraphobia; (c) Ranking of Labels of Specific Phobia; (d) Ranking of Labels of Social Phobia; (e) Ranking of Labels of Separation Anxiety Disorder; (f) Ranking of Labels of GAD; (g) Ranking of Labels of PTSD; (h) Ranking of Labels of OCD.

(a)

\begin{tabular}{|c|c|}
\hline Label Category & Participants \% \\
\hline Panic attacks & 27.8 \\
\hline Medical problem & 20.5 \\
\hline Anxiety & 12.9 \\
\hline Fear & 9.8 \\
\hline Other/non-specific ${ }^{*}$ & 8.5 \\
\hline No answer given & 6.9 \\
\hline Phobia & 2.5 \\
\hline Don’t know & 1.9 \\
\hline Worry & 1.9 \\
\hline Anxiety attacks & 1.6 \\
\hline Panic disorder & 1.3 \\
\hline Low confidence & 1.3 \\
\hline Nothing/no problem & 0.9 \\
\hline Paranoia & 0.9 \\
\hline Repeating the experience & 0.6 \\
\hline Trauma/Post traumatic stress & 0.6 \\
\hline Total & 100.0 \\
\hline
\end{tabular}

"Examples: "Shock", "careful to body", "Scared of own shadow".

(b)

\begin{tabular}{cc}
\hline Label Category & Participants \% \\
\hline Agoraphobia & 37.2 \\
Anxiety & 13.9 \\
Other/non-specific $^{*}$ & 10.1 \\
Phobia/anxiety of public places & 10.1 \\
Panic attacks & 6.9 \\
Fear/Worry & 6.3 \\
Other phobia & 4.1 \\
Social phobia/fear of people & 3.2 \\
Claustrophobia & 2.8 \\
No answer given & 1.9 \\
Paranoia & 1.3 \\
Anxiety disorder & 1.3 \\
Don't know & 0.6 \\
Nothing/no problem & 0.3 \\
Total & 100.0 \\
\hline
\end{tabular}

*Examples: "Cataplexy", "Psychological". (c)

\begin{tabular}{cc}
\hline Label Category & Participants \% \\
\hline Specific phobia & 33.4 \\
Fear/scared of storms & 16.4 \\
Other/non-specific ${ }^{*}$ & 13.9 \\
Anxiety & 12.6 \\
No answer given & 6.9 \\
Bad childhood experience & 5.0 \\
Paranoid & 4.7 \\
Anxiety disorder & 2.8 \\
Over-active imagination & 0.9 \\
Don’t know & 0.9 \\
Nothing/no problem & 0.9 \\
Panic attacks & 0.6 \\
Total & 0.6 \\
\hline
\end{tabular}

"Examples: "Hysteria", "Lack of knowledge", "Himself".

(d)

\begin{tabular}{cc}
\hline Label Category & Participants \% \\
\hline Shy & 26.8 \\
Low confidence/self esteem/insecure & 25.2 \\
Social phobia/social anxiety disorder & 10.7 \\
Other/non-specific ${ }^{*}$ & 9.8 \\
Socially awkward/social problems & 6.6 \\
Fear of social interaction & 6.0 \\
Anxiety & 5.4 \\
Other fear & 3.5 \\
No answer given & 1.6 \\
Don't know & 1.3 \\
Autism & 0.9 \\
Agoraphobia & 0.9 \\
Anxiety disorder & 0.6 \\
People phobia & 0.6 \\
Total & 100.0 \\
\hline
\end{tabular}

"Examples: "Doesn't want to do anything embarrassing", "stage fright", "inferiority complex". 
(e)

\begin{tabular}{|c|c|}
\hline Label Category & Participants \% \\
\hline Fear of losing parents/abandonment/separation & 14.2 \\
\hline Anxiety & 13.2 \\
\hline Other/non-specific ${ }^{*}$ & 11.7 \\
\hline Insecure & 9.5 \\
\hline Fear & 6.9 \\
\hline Worry & 6.3 \\
\hline Separation anxiety disorder & 6.0 \\
\hline Attachment problems & 4.4 \\
\hline No answer given & 4.4 \\
\hline Paranoid & 4.1 \\
\hline Stress & 2.5 \\
\hline Depression & 2.2 \\
\hline Past experience & 1.9 \\
\hline Fear of being alone & 1.6 \\
\hline Bullying & 1.6 \\
\hline Dependent on parents & 1.6 \\
\hline Obsessive disorder/OCD & 1.6 \\
\hline Don’t know & 1.6 \\
\hline Type of phobia & 1.3 \\
\hline $\mathrm{TV} /$ media contribution & 1.3 \\
\hline Nothing/no problem & 1.3 \\
\hline Anxiety disorder & 0.9 \\
\hline Total & 100.0 \\
\hline
\end{tabular}

"Examples: "Doesn't like idea of death", "Delusional", "Needs to man up".

(f)

\begin{tabular}{cc}
\hline Label Category & Participants \% \\
\hline Anxiety & 24.6 \\
Worrier/over-worries $^{*}$ (18.9 & 12.0 \\
Other/non-specific $^{*}$ & 9.1 \\
Stress & 7.9 \\
Insecure/lacks confidence/self-esteem & 4.4 \\
Depression & 4.4 \\
Obsessive/OCD & 3.2 \\
No answer given & 2.8 \\
GAD & 2.5 \\
Anxiety disorder & 2.2 \\
Sleep problems & 1.6 \\
Over-thinks & 1.6 \\
Nothing/no problem & 1.3 \\
Don't know & 1.3 \\
Paranoid & 1.3 \\
Nothing/no problem & 0.9 \\
Perfectionist & 100.0 \\
Total &
\end{tabular}

"Examples: "Himself", "PMT", "Indecisive". (g)

\begin{tabular}{cc}
\hline Label Category & Participants \% \\
\hline PTSD & 41.6 \\
Fear & 12.0 \\
Trauma from robbery/traumatized & 10.4 \\
Other/non-specific ${ }^{*}$ & 7.3 \\
Other relation to robbery & 6.3 \\
Anxiety & 5.4 \\
Sleep problems/Insomnia & 4.4 \\
No answer given & 3.8 \\
Nightmares & 1.9 \\
Stress & 1.6 \\
Paranoia & 1.3 \\
Panic & 1.3 \\
Depression & 1.3 \\
Agoraphobia & 0.9 \\
Don't know & 0.6 \\
Total & 100.0 \\
\hline
\end{tabular}

*Examples: "Intense worrying", "nervous", "fragile".

(h)

\begin{tabular}{cc}
\hline Label Category & Participants \% \\
\hline OCD & 64.7 \\
Phobia/fear of germs & 8.2 \\
Other/non-specific ${ }^{*}$ & 6.3 \\
Obsessive & 5.7 \\
Cleanliness/cleaning issues & 5.7 \\
Compulsive disorder & 4.4 \\
No answer given & 2.2 \\
Paranoid & 1.6 \\
Anxiety & 0.9 \\
Don't know & 0.3 \\
Total & 100.0 \\
\hline
\end{tabular}

"Examples: "Illness", "Repetitive".

cific phobia the lowest.

Ratings for whether participants should seek help from a GP or Psychologist were also compared across anxiety disorders; again testing the second part of the first hypothesis. Table 2 illustrates participants gave higher ratings for a Psychologist than a GP for all disorders except panic disorder. Two one-way repeated measures ANOVAs 
Table 2. Mean Ratings for Seeking Help from a GP and a Psychologist across the Anxiety Disorders.

\begin{tabular}{ccccc}
\hline & \multicolumn{2}{c}{ Psychologist } & \multicolumn{2}{c}{ GP } \\
\cline { 2 - 5 } & $\mathrm{M}$ & $\mathrm{SD}$ & $\mathrm{M}$ & $\mathrm{SD}$ \\
\hline Agoraphobia & 6.25 & 1.33 & 5.73 & 1.62 \\
PTSD & 6.22 & 1.2 & 5.56 & 1.77 \\
OCD & 6.18 & 1.38 & 5.49 & 1.74 \\
Specific phobia & 5.79 & 1.53 & 4.71 & 2.04 \\
Separation anxiety & 5.70 & 1.76 & 4.91 & 2.04 \\
disorder & & & & \\
Social phobia & 5.45 & 1.81 & 4.41 & 2.08 \\
GAD & 5.44 & 1.78 & 4.75 & 2.06 \\
Panic disorder & 4.91 & 2.01 & 5.89 & 1.66 \\
\hline
\end{tabular}

were conducted to determine whether there was a significant difference in ratings across the disorders. Results showed there was a significant difference in ratings for seeking help from a GP and a Psychologist across disorders. For Psychologist ratings the Mauchly test for sphericity was significant $(W=0.46, p<0.001)$ therefore with Greenhouse Geisser corrections the result was $F(5.82,1815.18)=48.21, p<0.001$. Similarly for GP rating, Mauchly's test was significant $(W=0.61, p<$ $0.001)$ and using Greenhouse Geisser, $F(6.02,1865.93)$ $=48.82, p<0.001$.

\subsection{Ratings for Perceived Life Adjustment}

One-way repeated-measure ANOVAs were carried out to test the second hypothesis, determining whether there was a significant difference between participants' ratings of how happy and successful at work, and how many satisfying personal relationships the character had, dependent on their anxiety disorder. Post-hoc pair-wise comparisons were then calculated; comparing the mean ratings for each three measures in order to find where the differences lay; this used Bonferroni adjustments to create a new significance level of 0.0018 .

For "happiness" ratings Mauchly's test of sphericity was significant ( $W=0.72, p<0.001)$. Using Greenhouse-Geisser, results showed that ratings of "happiness" were significantly different across anxiety disorders; $F(6.39,2012.04)=94.90, p<0.001$. Table 3 shows the means of the "happiness" ratings which are significantly different from each other; illustrating that characters with specific phobia, panic disorder and OCD are rated significantly "happier" than characters with all other disorders.

For "success at work" ratings Mauchly's test for sphericity was again significant $(W=0.72, p<0.001)$. Using Greenhouse-Geisser, results showed that the dif-
Table 3. Ranking of the Mean Rating for "Happiness" for each disorder.

\begin{tabular}{ccc}
\hline Disorders & $M$ & $S D$ \\
\hline Specific phobia & $4.09 \mathrm{a}$ & 1.34 \\
Whilst panic disorder & $3.81 \mathrm{~b}$ & 1.38 \\
OCD & $3.28 \mathrm{c}$ & 1.37 \\
GAD & $2.88 \mathrm{~d}$ & 1.35 \\
Separation anxiety disorder & $2.74 \mathrm{e}$ & 1.26 \\
Social phobia & $2.68 \mathrm{e}$ & 1.19 \\
PTSD & $2.58 \mathrm{e}$ & 1.18 \\
Agoraphobia & $2.42 \mathrm{f}$ & 1.15 \\
\hline
\end{tabular}

Means that share the same superscript are not significantly different from each other.

ferences in ratings of "success at work" were significantly different across anxiety disorders; $F(6.47,2019.23)$ $=94.41, p<0.001$. Table 4 shows the mean "success at work" ratings for each disorder and which are significantly different from each other. This found that characters with agoraphobia are rated significantly lower than all other disorders in "success at work", whereas specific phobia and panic disorder are rated the most successful, and significantly more so than other disorders.

For ratings of how "satisfying the characters personal relationships" were, Mauchly's test of sphericity was significant; $W=0.73, p<0.001$ and therefore GreenhouseGeisser estimates were used. The results indicated the ratings were significantly different across anxiety disorders; $F(6.43,2005.61)=99.49, p<0.001$. Table 5 shows the mean ratings for each anxiety disorder on how "satisfying personal relationships" the character has; illustrating which disorders are significantly different from each other. The comparisons found characters with social phobia were rated to have the least satisfying personal relationships, with a rating significantly lower than other disorders. Whilst panic disorder and specific phobia were rated significantly higher than other disorders; and said to have the most satisfying relationships.

An analysis was also conducted on the total adjustment of characters; calculated by an average score of the three adjustment ratings. Mauchly's test for sphericity was significant; $W=0.66, p<0.001$ and therefore Greenhouse-Geisser adjustments were used. The results indicated that overall adjustment ratings significantly differed across anxiety disorders; $F(6.22,1922.36)=$ 132.13, $p<0.001$. Table 6 shows the mean ratings for each anxiety disorder on total adjustment; illustrating which disorders were significantly different from each other. The post-hoc tests demonstrated that characters with specific phobia and panic disorder were seen as 
Table 4. Ranking of the Mean Rating for "Success at Work" for each disorder.

\begin{tabular}{ccc}
\hline Anxiety disorder & $M$ & $S D$ \\
\hline Specific phobia & $4.43 \mathrm{a}$ & 1.26 \\
Panic disorder & $4.29 \mathrm{a}$ & 1.24 \\
Social phobia & $3.63 \mathrm{~b}$ & 1.46 \\
OCD & $3.57 \mathrm{~b}$ & 1.53 \\
GAD & $3.51 \mathrm{~b}$ & 1.56 \\
Separation anxiety disorder & $3.03 \mathrm{c}$ & 1.23 \\
PTSD & $2.98 \mathrm{c}$ & 1.26 \\
Agoraphobia & $2.46 \mathrm{~d}$ & 1.34 \\
\hline
\end{tabular}

Means that share the same subscript are not significantly different from each other.

Table 5. Ranking of the Mean Rating for "Personal Relationships" for each disorder.

\begin{tabular}{ccc}
\hline Anxiety disorder & $M$ & $S D$ \\
\hline Specific phobia & $4.48 \mathrm{a}$ & 1.27 \\
Panic disorder & $4.39 \mathrm{a}$ & 1.20 \\
Separation anxiety disorder & $3.60 \mathrm{~b}$ & 1.44 \\
PTSD & $3.50 \mathrm{~b}, \mathrm{c}$ & 1.28 \\
OCD & $3.27 \mathrm{c} \mathrm{d}$ & 1.35 \\
GAD & $3.11 \mathrm{~d}$ & 1.26 \\
Agoraphobia & $3.04 \mathrm{~d}$ & 1.29 \\
Social phobia & $2.68 \mathrm{e}$ & 1.37
\end{tabular}

Means that share the same subscript are not significantly different from each other.

Table 6. Ranking of the Mean Rating for "Total Adjustment Score" for each disorder.

\begin{tabular}{ccc}
\hline Anxiety disorder & $M$ & $S D$ \\
\hline Specific phobia & $4.52 \mathrm{a}$ & 1.28 \\
Panic disorder & $4.41 \mathrm{a}$ & 1.22 \\
Separation anxiety disorder & $3.59 \mathrm{~b}$ & 1.46 \\
PTSD & $3.48 \mathrm{~b}$ & 1.28 \\
OCD & $3.30 \mathrm{~b}, \mathrm{c}$ & 1.35 \\
GAD & $3.11 \mathrm{c}$ & 1.27 \\
Agoraphobia & $3.02 \mathrm{c}$ & 1.30 \\
Social phobia & $2.69 \mathrm{~d}$ & 1.39 \\
\hline
\end{tabular}

Means that share the same subscript are not significantly different from each other. significantly better adjusted than others but not different from each other (although close to significance at the Bonferroni level, with $p=0.005$ ). Those with agoraphobia were significantly worse adjusted than all others. The ratings for the remaining disorders were not significantly different from the other disorders, with the exception of OCD to PTSD, to separation anxiety disorder and to social phobia. Although some were closer to the Bonferroni significance level of $p=0.0018$; GAD and $\mathrm{OCD}(p=$ $0.006)$ and social phobia and GAD $(p=0.015)$.

\subsection{Vignette Gender}

No significant effect of vignette gender on recognition of a disorder was found ( $p>0.2$ for all except specific phobia; $p=0.053$ ). To investigate whether vignette gender affected seeking help ratings, a non-parametric MannWhitney test was conducted; as the homogeneity of variance tests was significant for PTSD. Results did not find a significant effect of vignette gender on seeking help recommendations, with $p>0.4$. However PTSD was very close to significance $(p=0.051)$, with ratings for female vignettes higher than male vignettes $(M=6.58$ and 6.36 respectively).

To determine whether vignette gender had an effect on how sympathetic a participant would be towards someone with the problem, a one-way ANOVA was conducted. Results were not significant, showing no main effect of vignette gender on the sympathy rating given with most above $p=0.25$, though closer to significance was PTSD $(p=0.10)$. Despite the results not being significant, the mean sympathy rating was slightly higher for female versus male vignettes, except for cases of specific phobia, social phobia, separation anxiety disorder and GAD where male vignettes were given a higher sympathy rating.

\subsection{Participant Gender}

Females "correctly" identified disorders more, except for panic disorder. A significant difference was found for specific phobia $\left(X^{2}=12.87, p<0.001\right)$, PTSD $\left(X^{2}=\right.$ $14.52, p<0.001)$ and $\operatorname{OCD}\left(X^{2}=19.84, p<0.001\right)$.

Mann Whitney $U$ tests were conducted to test for an effect of participant gender on seeking help ratings. A significant effect of gender, with females giving significantly higher ratings for seeking help than males for $\operatorname{PTSD}(U=10845, Z=-2.57, p=0.01)$, separation anxiety disorder $(U=10684.5, Z=-2.48, p=0.013)$ and $\operatorname{OCD}(U=10887.5, Z=-2.27, p=0.024)$.

To test whether participant gender had an effect on adjustment ratings given, Mann Whitney $U$ tests were conducted. Males gave higher ratings of adjustment for disorders, except for panic disorder and specific phobia. Males gave significantly higher ratings for panic disorder 
$(U=8222.5, Z=-5.26, p<0.001)$, separation anxiety disorder $(U=9258.5, Z=-3.66, p<0.001)$, specific phobia $(U=9864, Z=-3.31, p=0.001)$, OCD $(U=$ $10734, Z=-2.23, p=0.026)$ and PTSD $(U=10941.5, Z$ $=-1.98, p=0.048)$. Tests were also conducted to determine whether there was an interaction between vignette gender and participant gender in response to ratings for seeking help and sympathy. No significant interactions were found, with $F<1.0$.

\section{DISCUSSION}

As predicted, levels of "correct" labelling varied significantly across disorders; with "correct" referring to using the currently accepted psychiatric term for the disorder. OCD had the highest recognition rates (64.67\%), and Figure 1 demonstrates that this was much higher than all other disorders, with a $23.03 \%$ difference between it and the second most "accurately" labelled disorder PTSD. OCD was recognised at a similar rate to depression [4] and schizophrenia [9], with the remaining anxiety disorders at a lower rate in comparison. PTSD, agoraphobia and specific phobia had relatively reasonable recognition rates; $41.64 \%, 37.54 \%$ and $34.07 \%$ respectively. However rates of "correct" recognition for social phobia and separation anxiety disorder were poor (10.73\% and 5.99\%) and for GAD and panic disorder were even worse $(2.84 \%$ and $1.26 \%)$. The data supports those who found higher recognition for OCD compared to GAD and panic disorder, though overall recognition rates were much lower in the current study perhaps due to the form of questioning [17]. The data also support others in recognition rates for social phobia; illustrating poor recognition of the disorder [14]. Levels of recognition for PTSD were similar to that in the above work, though slightly higher.

The results cannot explain why some ADs are more easily recognised than others. It could be that higher recognition rates for OCD, agoraphobia, PTSD and specific phobia, are easier to spot and less complex than the other disorders. It could be because they are discussed more in the media with various "celebrity cases" having these ADs. It does not however appear to relate to prevalence or incidence levels. Though the results when examining "correct" labelling suggest a relatively poor literacy for many anxiety disorders, the criterion for "correct" labelling was strict. When examining the other answers given by participants it is clear that people can recognise may symptoms of the disorders (Tables 1(a)(h)).

Most participants believed the person should seek help rather than not for all disorders. Specific phobia has the lowest rating for recommended treatment, with GAD close behind which could suggest people see these dis- orders as a normal part of everyday life. This would fit in with many of the labels given for GAD, such as $18.9 \%$ stating the character is a "worrier". Since most people experience worry and fear at some point, the public may not recognise these disorders as serious psychological problems, whereas the symptoms of PTSD and agoraphobia may be seen as more unusual and as having a greater impact on people, therefore explaining why they received higher help ratings.

Participants rated trained clinical Psychologists most highly in agoraphobia and PTSD. For panic disorder, the lowest rating was given to Psychologists but the highest for GPs (General Practioners/Physicians) which would suggest that participants saw the character for panic disorder more as a medical than a psychological problem; fitting in with $20.5 \%$ of people labelling it a "medical problem" and 27.8\% "panic attacks". However the very participants who labelled panic disorder "correctly" rated psychologists higher than a GP (6.75 compared to 2.5); which may suggest they recognised it more as a psychological problem and thus illustrating the benefit of "correct" labelling.

Variation in treatment beliefs may be partly due to a lack of knowledge about the disorders and their seriousness. However compared to recognition scores, the data does suggest a higher literacy concerning treatment, in the sense that relatively high ratings were given for seeking help regardless of the disorder.

The results supported the hypothesis (H1) that adjustment beliefs would vary significantly across the anxiety disorders; in ratings of happiness, success at work and how many satisfying personal relationships the vignette character had. People with panic disorder and specific phobia were judged to be significantly better adjusted than all other disorders. Those with Social Phobia were seen as the worst adjusted. It is likely that adjustment ratings vary across the disorders due to perceived seriousness of the different problems and symptoms which may prevent the character from living a "well-adjusted life". One interesting question that arises from this study would be the comparison of expert clinician judgements of adjustment compared to these judgments of young people.

Neither the gender of the vignette person with an $\mathrm{AD}$, nor the gender of the participants, was significant over all the ADs though there some differences on specific disorders. Overall, hypotheses $\mathrm{H} 2$ and $\mathrm{H} 3$ were not confirmed in the sense that there were sex differences across all the ADs although there were some small but significant differences, as predicted on individual ADs. The results therefore do not lend some support to that previously found on gender effects $[6,7,22]$. It has been argued that females having higher literacy in some cases may be because they are more aware of the disorders due 
to knowing they are more at risk of them; as has been demonstrated with higher prevalence rates [18]. Differences in adjustment scores due to gender, as found elsewhere [7] but not supported here, could suggest females have better literacy as they recognise the problems associated to disorders more than males and thus provide a lower adjustment score; though this was not true for panic disorder or specific phobia.

This study demonstrated that MHL of ADs is relatively poor with the exception of a good recognition rate of OCD. The results points towards the need for campaigns to increase knowledge about anxiety disorders; particularly panic disorder, GAD, separation anxiety disorder and social phobia. Past interventions to improve MHL have had successes, with campaigns increasing literacy levels and leading to improved help seeking [30]. Many websites are now available for information on mental health; three sites focused on anxiety disorders were reviewed by researchers, who concluded they were generally poor and inadequate [31].

This study had various limitations. Vignette methodology has various problems particularly the comparative clarity, representativeness and severity of the different vignettes.

Small changes can have significant consequences for the results and therefore this study merits replication with different vignettes. It would, ideally, have been better to have more than one vignette of the same disorder. Next, although this was a reasonably sized sample it was biased towards better educated people and those more familiar with mental illnesses than the general population.

This would suggest, if anything, the general population is more poorly informed about the ADs than this study would suggest. However to ensure the generalizability of these results it would have been preferable to have a larger, more representative sample.

\section{REFERENCES}

[1] Jorm, A., Korten, A., Jacomb, P., Christensen, H., Rodgers, B. and Politt, P. (1997) "Mental health literacy": A survey of the public's ability to recognise mental disorder and their beliefs about the effectiveness of treatment. $\mathrm{Me}$ dical Journal of Australia, 166, 182-186.

[2] Jorm, A. (2012) Mental health literacy. American Psychologist, 67, 231-243. doi:10.1037/a0025957

[3] Furnham, A. and Blythe, C. (2012) Schizophrenia literacy. Psychiatric Research, 198, 18-23. doi:10.1016/j.psychres.2011.12.025

[4] Wang, J., Adair, C., Fick, G., Lai, D., Perry, B.W., Jorm, A. and Addington, D. (2007) Depression literacy in Alberta: Findings from a general population sample. Canadian Journal of Psychiatry, 52, 442-449.

[5] Furnham, A. and Carter-Leno, V. (2012) Psychiatric lit- eracy and the conduct disorders. Research in Developmental Disability, 33, 24-31.

doi:10.1016/j.ridd.2011.08.001

[6] Cotton, S., Wright, A., Harris, M., Jorm, A. and McGorry, P. (2006) Influence of gender on mental health literacy in young Australians. Australian and New Zealand Journal of Psychiatry, 40, 790-796. doi:10.1080/i.1440-1614.2006.01885.x

[7] Furnham, A., Cook, R., Martin, N. and Batey, M. (2011) Mental health literacy among university students. Journal of Public Mental Health, 10, 198-210. doi:10.1108/17465721111188223

[8] Leighton, S. (2009) Adolescent's understanding of mental health problems. Journal of Public Health, 8, 4-14.

[9] Furnham, A., Daoud, Y. and Swami, V. (2009) "How to spot a psychopath": Lay theories of psychopathy. Social Psychiatry and Psychiatric Epidemiology, 44, 464-472. doi:10.1007/s00127-008-0459-1

[10] American Psychiatric Association DSM-5 Development (2012) Anxiety disorders. Retrieved 22February 2012. http://www.dsm5.org/proposedrevision/Pages/AnxietyDis orders.aspx

[11] Michael, T., Zetsche, U. and Margraf, J. (2007) Epidemiology of anxiety disorders. Psychiatry, 6, 136-142. doi:10.1016/j.mppsy.2007.01.007

[12] Kessler, R., Chiu, W., Demler, O. and Walters E. (2005) Prevalence, severity, and comorbidity of twelve-month DSM-IV disorders in the National Comorbidity Survey Replication (NCS-R). Archives of General Psychiatry, 62 , 617-627. doi:10.1001/archpsyc.62.6.617

[13] Merikangas, K., He, J., Burstein, M., Swanson, S., Avenevoli, S., Cui, L., Benjet, C., Georgiades, K. and Swendsen, J. (2010). Lifetime prevalence of mental disorders in US adolescents: Results from the national co-morbidity survey replication-Adolescent Supplement (NCS-A). Journal of the American Academy of Child \& Adolescent Psychiatry, 49, 980-989. doi:10.1016/i.jaac.2010.05.017

[14] Reavley, N. and Jorm, A. (2011) Recognition of mental disorders and beliefs about treatment and outcome: Findings from an Australian national survey of mental health literacy and stigma. Australian and New Zealand Journal of Psychiatry, 45, 947-956. doi: $10.3109 / 00048674.2011 .621060$

[15] Munro, C., Freeman, C. and Law, R. (2004) General practitioners' knowledge of post-traumatic stress disorder: A controlled study. British Journal of General Practice, 54, 843-847.

[16] Ehlers, A., Gene-Cos, N. and Perrin, S. (2009) Low recognition of post-traumatic stress disorder in primary care. London Journal of Primary Care, 2, 36-42.

[17] Coles, M.E. and Coleman, S.L. (2010) Barriers to treatment seeking for anxiety disorders: Initial data on the role of mental health literacy. Depression and Anxiety, 27, 6371. doi: $10.1002 /$ da. 20620

[18] McLean, C., Asnaani, A., Litz, B. and Hofmann, S. (2011) Gender differences in anxiety disorders: Prevalence, course of illness, comorbidity and burden of illness. Journal of 
Psychiatric Research, 45, 1027-1035. doi:10.1016/j.jpsychires.2011.03.006

[19] Xu, Y., Schneier, F., Heimberg, R., Princisvalle, K., Liebowitz, M., Wang, S. and Blanco, C. (2012) Gender differences in social anxiety disorder: Results from the national epidemiologic sample on alcohol and related conditions. Journal of Anxiety Disorders, 26, 12-19. doi:10.1016/i.janxdis.2011.08.006

[20] Fan, F., Zhang, Y., Yang, Y., Mo, L. and Liu, X. (2011) Symptoms of posttraumatic stress disorder, depression, and anxiety among adolescents following the 2008 Wenchuan earthquake in China. Journal of Traumatic Stress, 24, 44-53. doi:10.1002/jts.20599

[21] Dell'Osso, L., Carmassi, C., Massimetti, G., Daneluzzo, E., Di Tommaso, S. and Rossi, A. (2011) Full and partial PTSD among young adult survivors 10 months after the L'Aquila 2009 earthquake: Gender differences. Journal of Affective Disorders, 131, 79-83. doi:10.1016/j.jad.2010.11.023

[22] Wong, D., Lam, A., Poon, A. and Chow A. (2011) Gender differences in mental health literacy among Chinese-speaking Australians in Melbourne, Australia. International Journal of Social Psychiatry, 42, 1-8.

[23] Bener, A. and Ghuloum, S. (2010) Gender differences in the knowledge, attitude and practice towards mental health illness in a rapidly developing Arab society. International Journal of Social Psychiatry, 57, 480-486. doi:10.1177/0020764010374415

[24] Wright, A., Jorm, A., Harris, M. and McGorry, P. (2007)
What's in a name? Is accurate recognition and labelling of mental disorders by young people associated with better help-seeking and treatment preferences? Social Psychiatry and Psychiatric Epidemiology, 42, 244-250. doi:10.1007/s00127-006-0156-X

[25] Wright, A., Jorm, A. and Mackinnon, A. (2011) Labelling of mental disorders and stigma in young people. Social Science \& Medicine, 73, 498-506. doi:10.1016/j.socscimed.2011.06.015

[26] Herran, A., Vázquez-Barquero, J., Dunn, G., Johnston, T. and Talbot, P. (1999) Recognition of depression and anxiety in primary care. British Medical Journal, 318, 15581559. doi:10.1136/bmj.318.7197.1558

[27] Seligman, M., Walker, E. and Rosenhan, D. (2001) Abnormal psychology. Norton \& Company Inc.

[28] Galanter, C. and Jensen, P. (2009) DSM-IV-TR casebook and treatment guide for child mental health. American Psychiatric Publishing Inc., Washington DC, 43-44.

[29] Spitzer, R., Gibbon, M., Skodol, A., Williams, J. and First, M. (2002) DSM-IV-TR case book. American Psychiatric Publishing Inc., Washington DC.

[30] Jorm, A. (2000) Mental health literacy: Public knowledge and beliefs about mental disorders. British Journal of Psychiatry, 177, 396-401. doi:10.1192/bjp.177.5.396

[31] Reavley, N. and Jorm, A. (2011). The quality of mental disorder information websites: A review. Patient Education and Counselling, 85, 16-25. doi:10.1016/j.pec.2010.10.015 